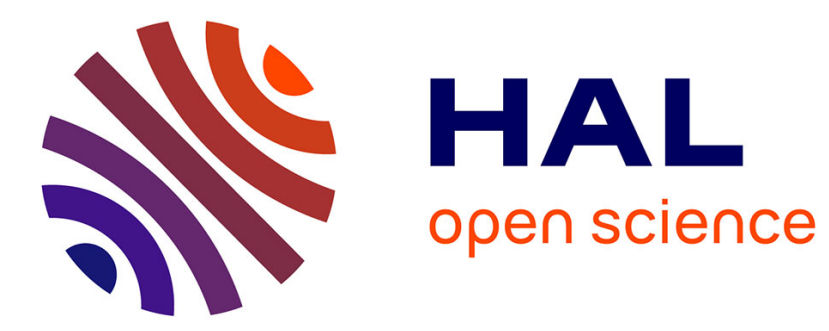

\title{
Exotic Freezing of Response in a Quantum Many-body System
}

Arnab Das

\section{To cite this version:}

Arnab Das. Exotic Freezing of Response in a Quantum Many-body System. Physical Review B: Condensed Matter (1978-1997), 2010, 82, pp.172402. 10.1103/PhysRevB.82.172402 . hal-03019447

\section{HAL Id: hal-03019447 \\ https://hal.science/hal-03019447}

Submitted on 23 Nov 2020

HAL is a multi-disciplinary open access archive for the deposit and dissemination of scientific research documents, whether they are published or not. The documents may come from teaching and research institutions in France or abroad, or from public or private research centers.
L'archive ouverte pluridisciplinaire HAL, est destinée au dépôt et à la diffusion de documents scientifiques de niveau recherche, publiés ou non, émanant des établissements d'enseignement et de recherche français ou étrangers, des laboratoires publics ou privés. 


\title{
Exotic freezing of response in a quantum many-body system
}

\author{
Arnab Das \\ The Abdus Salam International Centre for Theoretical Physics (ICTP), Trieste 34151, Italy and Theoretical Division (T-4), LANL, \\ MS-B213, Los Alamos, New Mexico 87545, USA
}

(Received 12 August 2010; revised manuscript received 20 September 2010; published 3 November 2010)

\begin{abstract}
We show that when a quantum many-body system is subjected to coherent periodic driving, the response may exhibit exotic freezing behavior in high driving frequency $(\omega)$ regime. In a periodically driven classical thermodynamic system, freezing at high $\omega$ occurs when $1 / \omega$ is much smaller than the characteristic relaxation time of the system and hence the freezing always increases there as $\omega$ is increased. Here, in the contrary, we see surprising nonmonotonic freezing behavior of the response with $\omega$, showing curious peak-valley structure. Quite interestingly, the entire system tends to freeze almost absolutely (the freezing peaks) when driven with a certain combination of driving parameters values (amplitude and $\omega$ ) due to coherent suppression of dynamics of the quantum many-body modes, which has no classical analog. We demonstrate this new freezing phenomenon analytically (supported by large-scale numerics) for a general class of integrable quantum spin systems.
\end{abstract}

DOI: $10.1103 /$ PhysRevB.82.172402

PACS number(s): 75.40.Gb, 03.65.Vf, 05.30.-d, 42.50.Ar

The field of driven dynamics in quantum many-body system has attracted a lot of theoretical attention in last few years (see, e.g., Refs. 1-9). The major part of this research concentrated mainly around slow quenching dynamics across quantum-critical points (lines/surfaces) resulting in quantum Kibble-Zurek mechanism (KZM) (Refs. 2-4) of generating scaling laws for defect densities-a direct translation of the consequences of robust classical KZM (Ref. 10) in the quantum regime. The distinctive role of quantum coherence in driven many-body dynamics thus somehow remained still largely unexplored (see, however, Refs. 8 and 11-13). Experimentally, on the other hand, this coherent regime is becoming much more accessible in recent years, thanks to the breakthrough in realizing highly isolated many-body quantum systems with long coherence time within the setup of cold atom in optical lattice (see, e.g., Ref. 14).

In this Brief Report we report an early attempt to explore this regime, studying the Schrödinger dynamics of a class of integrable quantum spin systems. The study reveals two generic regimes of the driving frequency $\omega$ : in the large $\omega$ regime (defined later), we observe surprising nonmonotonic behavior of the response with respect to $\omega$, showing peak and valley structures, which dramatically contrasts the expected monotonic behavior ubiquitous in the corresponding classical scenarios of periodic driving (say, where a classical magnet is driven externally by a time-periodic magnetic field at finite $T) \cdot{ }^{10,15-17,19}$ Here, for certain combinations of driving parameters, $\left(\omega\right.$ and the driving amplitude $h_{0}$ ) the entire many-body dynamics freezes almost absolutely giving rise to spectacular peaks. In the low $\omega$ regime, however, the peaks smooth out and a (roughly) monotonic behavior emerges as expected. We illustrate the crucial role of quantum coherence behind the phenomenon. To clarify the analogy, we may note that in a driven classical thermodynamic system, "following the driving field" means following the trail of instantaneous thermal equilibrium states corresponding to the instantaneous values of the time-varying driving field, where as for a quantum system at $T=0$, this means following the instantaneous ground state of the time-varying Hamiltonian.

In the classical case, faster driving always tends to leave the response more frozen in a monotonic manner in the high $\omega$ regime. The rationale is: a faster driving would allow lesser time for the system to react and hence the response would be left more frozen. The most universal and successful theory for non-equilibrium response behavior (e.g., defect formation) in a driven classical system, namely, the KZM, is based on this rationale. ${ }^{10}$ The quantum version of KZM (Refs. 2-4) is also based on the same classical notion of freezing-the response of a driven system gets "frozen" when its instantaneous relaxation rate falls below the driving rate. The response therefore remains frozen over a finite region around the critical point, where this condition is met. The faster the driving rate is, the larger is this region of freezing and thus more frozen is the response (e.g., for linear driving across a critical point, the said region of freezing increases as a power of the driving rate, the power being given by the static critical exponents ${ }^{4,10}$ ).

Though the above classical rationale leads to the correct trend of the freezing (the scaling law) even for the quantum systems in the small $\omega$ regime, here we demonstrate that it may surprisingly fail in some other cases when $\omega$ is high enough. In this regime, additional freezing may occur due to dynamics-dependent coherent cancellation of transition amplitude. We derive closed-form analytical expression for the entire nonmonotonic profile of the response, which accurately reproduces the (directly integrated) numerical results for large system size $\left(N=10^{4}\right)$. The dependence of the response on the amplitude of the driving field is also shown to exhibit trend completely reverse of that observed with incoherent classical fluctuations. We demonstrate this quantum freezing phenomena for a general class of integrable $d$-dimensional quantum system with Hamiltonians of the form (in momentum space)

$$
\mathcal{H}(t)=\sum_{\vec{k}} \psi_{\vec{k}}^{\dagger}\left(\begin{array}{cc}
h_{z}(t)+f_{\vec{k}} & \Delta_{\vec{k}} \\
\Delta_{\vec{k}}^{*} & -h_{z}(t)-f_{\vec{k}}
\end{array}\right) \psi_{\vec{k}},
$$

where $\psi_{\vec{k}}=\left(c_{1 \vec{k}}, c_{2 \vec{k}}\right)$ are standard fermionic operators in $\vec{k}$ space, $h_{z}(t)=h_{0} \cos (\omega t)$ is the driving field (any Hamiltonian parameter) and $f_{\vec{k}}$ (real) and $\Delta_{\vec{k}}$ are system-specific functions. The above-mentioned class includes many well-known quan- 

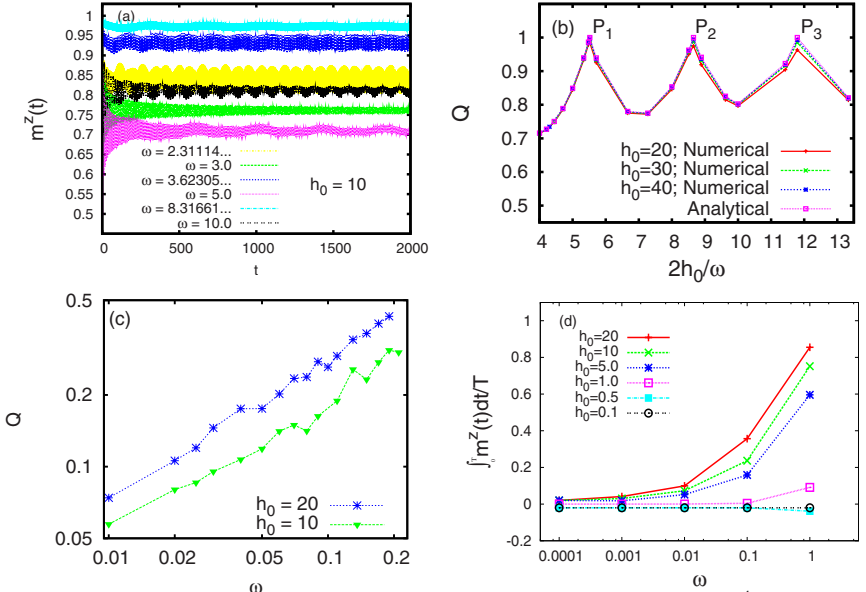

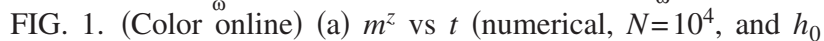
=10) for various $\omega \mathrm{s}$. (b) $Q$ vs $2 h_{0} / \omega$ for $h_{0}=20,30$, and 40, compared with the analytical formula $Q=\frac{1}{1+\left|J_{0}\left(2 h_{0} / \omega\right)\right|}$ [Eq. (7)]. The peaks $P_{1}, P_{2}$, and $P_{3}$ representing maximal freezing, corresponds to three zeros of $J_{0}\left(\frac{2 h_{0}}{\omega}\right)$, occurring at $\frac{2 h_{0}}{\omega}$ $=5.520, \ldots, 8.653, \ldots, 11.971, \ldots$, respectively. (c) $Q$ vs $h_{0}$ for $\omega$ $\ll 1$ (numerical). The peaks are smoothed out yielding a roughly monotonic behavior. (d) The counterclassical trend of stronger freezing for higher $h_{0}$ is demonstrated with single-sweep results; we plot $Q_{s}=\frac{1}{T} \int_{0}^{T} m^{z}(t) d t$, (where $\left.T=2 \pi / \omega\right)$ for $N=100$. The figure also demonstrates monotonic adiabatic to nonadiabatic transition at low $\omega$ regime for different $h_{0}$.

tum spin models in one, two and three dimensions, such as the transverse field Ising model (TFIM), quantum X-Y model, and extended Kitaev models (see Refs. 5 and 18 and references therein). We focus on TFIM for concrete illustration of the phenomenon because of its intuitive appeal but keep the calculation general so that the main result is easily visible for all the above-mentioned models. For TFIM with Hamiltonian

$$
H(t)=-\frac{1}{2}\left[J \sum_{i=1}^{N} \sigma_{i}^{x} \sigma_{i+1}^{x}+h_{z}(t) \sum_{i=1}^{N} \sigma_{i}^{z}\right],
$$

$\left[h_{z}(t)=h_{0} \cos (\omega t)\right.$ is the driving field and $\sigma_{i}^{x / z}$ are $x / z$ component of the Pauli spin], the form in Eq. (1) with $f_{\vec{k}}$ $=J \cos k$ and $\Delta_{\vec{k}}=J \sin k$ is obtained via Jordan-Wigner transformation followed by Fourier transform. If one starts with the ground state of the Hamiltonian at $t=0$, the timedependent wave function $|\psi(t)\rangle$ for the system may be expressed as a direct product of two-dimensional timedependent wave functions: $|\psi(t)\rangle=\otimes_{k>0}\left|\psi_{k}(t)\right\rangle$ with $\left|\psi_{k}(t)\right\rangle$ $=u_{\vec{k}}(t)\left|0_{+k} 0_{-k}\right\rangle+v_{\vec{k}}(t)|+k,-k\rangle$, where $\left|0_{+k} 0_{-k}\right\rangle$ and $|+k,-k\rangle$ represent, respectively, the unoccupied and the doubly occupied states of the $\pm k$ fermions. We start at $t=0$ from the ground state of the Hamiltonian $H(t=0)$ in Eq. (2) with $h_{0}$ $\gg 1$ (highly polarized in $+z$ direction) and as the field oscillates we measure the corresponding response function, the transverse magnetization

$$
m^{z}(t)=\left\langle\psi(t)\left|\sigma_{i}^{z}\right| \psi(t)\right\rangle=\frac{4}{N_{k}} \sum_{k 00}\left|v_{\vec{k}}(t)\right|^{2}-1 .
$$

The resulting time evolution of $m^{z}$, obtained by numerical integration of the time-dependent Schrödinger equation
(TDSE) for many sweeps, is shown in Fig. 1(a), for different $\omega$ 's. In each case, $m^{z}$ is found to remain confined within a narrow range in the positive sector (starting with $m^{z} \approx 1$ ) for all time, though the field $h_{z}$ oscillates symmetrically about zero. The dynamical symmetry breaking (due to freezing near the initial state) is quantified by the so-called dynamical order parameter $Q$, which is the long-time average of the response function. ${ }^{15}$ For TFIM

$$
Q=\left\langle m^{z}\right\rangle=\lim _{T_{f} \rightarrow \infty} \frac{1}{T_{f}} \int_{0}^{T_{f}} m^{z}(t) d t .
$$

Dynamical symmetry breaking $(Q \neq 0)$ is shown for different values of $\omega$ and $h_{0}$ in Figs. 1(a) and 1(b).

The intuitive reason for nonzero $Q$ might simply be the lack of adiabaticity in the dynamics. Nonadiabaticity occurs when the characteristic response time of a quantum system, given by the inverse of the relevant energy gap, is large compared to the driving period. Thus freezing due to the competition of these two time scales (the basis of quantum KZM estimate ${ }^{2-4}$ ) is similar in spirit (though very different in mechanism) with the classical dynamical hysteresis (freezing of the magnetization dynamics in a classical magnet when driven too fast by a periodic magnetic field). The similarity is only in the sense, that both freezing represent the failure of the system's reflex to adjust to the rapidly changing field, and hence in both cases stronger freezing is expected as the driving is made faster. In the classical case, the freezing is in fact always monotonic with respect to $\omega$. For example, in case of a periodically driven classical Ising model, ${ }^{15}$ the frozen (asymmetric; $Q \neq 0$ ) phase shrinks monotonically in the symmetric-asymmetric phase diagram as $\omega$ is reduced and finally vanishes as $\omega \rightarrow 0$. This monotonicity is also observed even here, when $\omega$ is small enough, as shown in Fig. 1(d). Surprisingly, contrary to this picture, we find $Q$ to be a nonmonotonic function of $\omega$, exhibiting peaks appearing at high $\omega$ 's as shown in Fig. 1(b). The peaks represent maximal freezing of the system with $Q$ very close to unity, indicating additional freezing for certain combinations of $\omega$ and $h_{0}$. In the low-frequency regime, however, the peaks are found to be smoothed out (roughly) and $Q$ is observed to decrease more or less monotonically with $\omega$ as shown in Fig. 1(c). In analogy with the classical case, we call this nonmonotonic quantum freezing phenomenon (and its related dynamical symmetry breaking) dynamical quantum hysteresis (DQH).

We explain the above scenario solving the dynamics of the $k$ modes as follows. Employing the $2 \times 2$ unitary transformation $\hat{U}_{k}=\exp \left[-\frac{i}{2}\left(2 t f_{\vec{k}}+\frac{2 h_{0}}{\omega} \sin \omega t\right) \sigma^{z}\right]$ on $\left|\psi_{k}(t)\right\rangle$ and $H_{k}(t)$ and performing a subsequent expansion in terms of Bessel's functions: $\exp [i z \sin \theta]=\sum_{n=-\infty}^{+\infty} J_{n}(z) e^{i n \theta}\left(J_{n}(z)\right.$ being the Bessel's function of the first kind with integer order $n),{ }^{20}$ we get the transformed wave function $\left|\psi_{k}^{\prime}(t)\right\rangle$ $=u_{\vec{k}}^{\prime}(t)\left|0_{+k}, 0_{-k}\right\rangle+v_{\vec{k}}^{\prime}(t)|+k,-k\rangle$ that follows the TDSE with the transformed Hamiltonian: 


$$
H_{k}^{\prime}(t)=-\Delta_{\vec{k}}\left(\begin{array}{cc}
0 & i \sum_{n=-\infty}^{\infty} R_{n} \\
-i \sum_{n=-\infty}^{\infty} R_{n}^{*} & 0
\end{array}\right) \text {, }
$$

where $R_{n}=J_{n}\left(\frac{2 h_{0}}{\omega}\right) e^{-i\left(n \omega+2 f_{\vec{k}}\right) t}$. We note that $\left|u_{\vec{k}}^{\prime}(t)\right|^{2}=\left|u_{\vec{k}}(t)\right|^{2}$ and $\left|v_{\vec{k}}^{\prime}(t)\right|^{2}=\left|v_{\vec{k}}(t)\right|^{2}$. Now we resort to the resonance approximation (RA), under which the equation are soluble (see, e.g., Ref. 21). The RA amounts to ignoring all the (faster oscillating) terms in the off-diagonal sum in $H_{k}^{\prime}(t)$ except for the resonant term $n=n_{r}$, for which the effective frequency $\Omega_{k}=\left|n \omega+2 f_{\vec{k}}\right|$ is the smallest. In the high frequency limit $\left(\omega \gg 2\left|f_{\vec{k}}\right|\right)$, we have $n_{r}=0$. Physically, this means, $\omega$ is far off-resonant with the relevant characteristic frequencies of the system given by $2 J \cos k(J=1$ here). For the general $d$-dimensional Hamiltonian (1), the criteria reads $\omega \gg\left|f_{\vec{k}}\right|$. The general solution of the TDSE with Hamiltonian (5) under RA gives

$$
\begin{aligned}
& v_{\vec{k}}(t)=-2 i e^{i f_{\vec{k}} t}\left[\frac{J_{0}\left(2 h_{0} / \omega\right) \Delta_{\vec{k}}}{2 \phi_{\vec{k}}} \sin \left(\phi_{\vec{k}} t\right)\right] u_{\vec{k}}(0)+e^{i f_{\vec{k}} t}\left[\cos \left(\phi_{\vec{k}} t\right)\right. \\
& \left.-i \frac{f_{\vec{k}}}{\phi_{\vec{k}}} \sin \left(\phi_{\vec{k}} t\right)\right] v_{\vec{k}}(0) \text {, }
\end{aligned}
$$

where $\phi_{\vec{k}}=\sqrt{J_{0}^{2}\left(2 h_{0} / \omega\right) \Delta_{\vec{k}}^{2}+f_{\vec{k}}^{2}}$. With the initial condition mentioned above $\left(\left|v_{\vec{k}}(0)\right|^{2} \approx 1\right)$, one gets

$$
\left|v_{\vec{k}}(t)\right|^{2}=1-\frac{J_{0}^{2}\left(2 h_{0} / \omega\right) \Delta_{\vec{k}}^{2}}{J_{0}^{2}\left(2 h_{0} / \omega\right) \Delta_{\vec{k}}^{2}+f_{\vec{k}}^{2}} \sin ^{2}\left(\phi_{\vec{k}} t\right) .
$$

From Eq. (6) we see that for the near-critical modes $(k$ $\left.\sim 0, \pi, \Delta_{\vec{k}} \sim 0\right),\left|v_{\vec{k}}(t)\right|^{2}$ oscillates with a vanishing amplitude proportional to $\Delta_{\vec{k}}^{2}$, and thus contribute maximally to the freezing. On the other hand, the off-critical modes $(k$ $\sim \pi / 2$ ) undergo full oscillation without any appreciable freezing. The intermediate modes, $\left(1>\left|\Delta_{\vec{k}}\right| \gg 0\right)$ oscillating with an amplitude that depend both on $k$ and the ratio $h_{0} / \omega$ [Eq. (6)] contribute nontrivially to the freezing. Nonmonotonic freezing is encoded here in the nonmonotonicity of $J_{0}(x)$. Any local observable is obtained by summing up the contributions from all these nonlocal many-body modes. To get an explicit formula for $Q$ for TFIM, we set $\Delta_{\vec{k}}=\sin k$, $f_{\vec{k}}=\cos k$, and take the continuum limit of Eq. (3). Integrating over $k$, taking the limit $T_{f} \rightarrow \infty$ of Eq. (4) gives a simple formula

$$
Q=\frac{1}{1+\left|J_{0}\left(2 h_{0} / \omega\right)\right|} .
$$

The expression matches remarkably well with the peakedstructured profile of $Q$ obtained by numerical integration, as shown in Fig. 1(b). The peaks occur for certain combinations of $\omega$ and $h_{0}$, for which $J_{0}\left(\frac{2 h_{0}}{\omega}\right)=0$. Under this condition, all the modes freeze, resulting in an absolute localization (within the RA approximation made) of the system at its initial state for all time (known as coherent destruction of tunneling in the context of driven two-level system ${ }^{22}$ ). The exact form of $Q$ depends on the model dimension and other system-specific details but the key feature-nonmonotonicity is already reflected in the general equation, Eq. (6).

To clarify the fundamental difference between the nature of this additional freezing and the freezing due to KZM, we note that after the first sweep the nonadiabatic excitation probability $p_{k}=\left|v_{k}(\omega t=\pi)\right|^{2}$ for each mode [related both to the total defect density ${ }^{24}$ and $m^{z}$, Eq. (3)], is actually a nonmonotonic function of $\omega$ [Eq. (6)]. In contrast, KZM would predict in such cases, a monotonic increase in the size of the impluse region $(2 \hat{\boldsymbol{\epsilon}})$ with $\omega$, resulting in a monotonically increasing $p_{k}$ (see, e.g., Ref. 4). This monotonicity is a general charactersistic of KZM as long as $|\dot{\boldsymbol{\epsilon}}|$ is either constant or increases monotonically as the critical point is approached. ${ }^{10}$

In the regime $\omega \ll\left|f_{\vec{k}}\right|$, however, the off-diagonal sum in $H_{k}^{\prime}(t)$ cannot be approximated by a single-frequency term. Presence of many close multiples of $\omega$ satisfying resonance condition $\Omega_{k}=\left|n \omega+2 f_{\vec{k}}\right| \sim 0$, smooths out the peaks and a gross monotonic behavior emerges, as shown in Fig. 1(c). The dynamics remain nonadiabatic due to quantum-critical points at $h_{z}= \pm 1$ for any nonzero $\omega$ in the thermodynamic limit. A more detailed study of the low-frequency behavior will be reported elsewhere. ${ }^{23}$

Phase coherence plays a crucial role in determining $Q$, as can be seen from the following example. In the limit $h_{0}$ $\gg|\sin (k)|$, the evolution corresponding to a full driving cycle might be decomposed into adiabatic and impulse regimes, 2,20 such that apart from some neighborhood $\pm \Delta h$ of the critical points at $h_{c}= \pm 1$, the dynamics is adiabatic, while within these neighborhoods the dynamics is impulsive, and can be approximated by Landau-Zener transitions upon linearizing the sinusoidal field for low enough $\omega$. Now, if the phase coherence between the fermionic state $\left|0_{+k}, 0_{-k}\right\rangle$ and $v_{\vec{k}}^{\prime}(t) \mid$ $+k,-k\rangle$ is neglected (see, for example, Ref. 24), say, due to some decoherence mechanism, then the fermionic excitations after $n$ complete cycles would be given by $\left|v_{\vec{k}}(n T)\right|^{2}=\frac{1}{2}[1$ $\left.+\left(2 \theta_{k}-1\right)^{n}\right]$, where $\theta_{k}=\exp \left[-\frac{\pi \sin ^{2}(k)}{\omega \sqrt{h_{0}^{2}-\cos ^{2} k}}\right]$. This implies, $m^{z}$ approaches 0 rapidly, giving $Q=0 \forall \omega$, contrasting the coherent case results [Fig. 1(b)].

The behavior of $Q$ with $h_{0}$ also contrasts the classical picture in a drastic way. In the classical case of a periodically driven magnet in presence of thermal fluctuations, dynamical localization always occurs below a certain value of the amplitude $h_{0}$ (for a given $\omega$ and temperature), above which the symmetric phase appears. ${ }^{15,17}$ High-enough driving fields in a classical Ising magnet (even in quantum magnets with some coherence) kills any hysteresis/freezing, forcing the system more strongly to follow the field as demonstrated experimentally by Aeppli's group. ${ }^{25}$ But in DQH, just the reverse trend is observed, as shown in Fig. 1(d) (low $\omega$ regime). In the high $\omega$ limit also, one has $J_{0}(x) \approx \sqrt{\frac{2}{\pi x}} \cos (x$ $-\pi / 4)$ for $x \gg \frac{1}{4}$ and the expression (7) reduces to

$$
Q \approx 1-\frac{\sqrt{\omega} \cos \left(\frac{2 h_{0}}{\omega}-\frac{\pi}{4}\right)}{\sqrt{\pi h_{0}}+\sqrt{\omega} \cos \left(\frac{2 h_{0}}{\omega}-\frac{\pi}{4}\right)},
$$

with $\lim _{h_{0} \rightarrow \infty} Q=1$, giving absolute freezing of the dynamics in this limit. A general qualitative explanation of this reverse 
trend goes as follows. In dynamics driven by classical fluctuations, a stronger field would induce stronger asymmetry between the rate of the aligning (spins orienting parallel to the field) and the antialigning dynamics, favoring the former one energetically over the latter. Hence for a higher $h_{0}$, the spins would realign faster along the field following a field reversal and thus the hysteresis/freezing would be reduced. But in the case of coherent quantum fluctuations, a stronger field would instead, more strongly suppress all the dynamics that would change the response, even if it helps lowering the field-induced potential enery, since the response (by definition) commutes with the field part of the Hamiltonian.

Experimental observation of the $\mathrm{DQH}$ phenomenon may be realizable in several ways. First, realization of this phenomenon would be possible in tunable transverse Ising model using trapped ions. ${ }^{26} \mathrm{~A}$ similar realization would be possible within lattice-spin models with polar molecules on optical lattices. ${ }^{27}$ In these systems, the exchange interaction $J$ have experimental upper limits $(\sim 22.1 \mathrm{kHz}$ and between $10-100 \mathrm{kHz}$ for the respective cases mentioned above) but can be made arbitrarily small. Hence the range of high $\omega$ referred here (in the units of $J$ ) may be brought down to a comfortable range, say to the order of few $\mathrm{kHz}$ in both the realizations. The phenomenon of $\mathrm{DQH}$ in Kitaev models ${ }^{18}$ can be achieved via experimental setups as proposed in Ref. 28. Its implication in the context of quantum annealing, ${ }^{29}$ might be quite interesting, as it may contrast the intuitive scenario of monotonic improvement with slower annealing in certain cases.

The author thanks B. K. Chakrabarti, G. Santoro, E. Tosatti, K. Sengupta, W. H. Zurek, B. Damski, N. Surendran, and A. Silva for useful discussions.
${ }^{1}$ A. Das et al., Phys. Rev. B 74, 144423 (2006).

${ }^{2}$ B. Damski, Phys. Rev. Lett. 95, 035701 (2005).

${ }^{3}$ W. H. Zurek, U. Dorner, and P. Zoller, Phys. Rev. Lett. 95, 105701 (2005).

${ }^{4}$ J. Dziarmaga, Phys. Rev. Lett. 95, 245701 (2005); Adv. Phys. 59, 1063 (2010).

${ }^{5}$ K. Sengupta, D. Sen, and S. Mondal, Phys. Rev. Lett. 100, 077204 (2008); D. Sen, K. Sengupta, and S. Mondal, ibid. 101, 016806 (2008).

${ }^{6}$ D. Patanè, A. Silva, L. Amico, R. Fazio, and G. E. Santoro, Phys. Rev. Lett. 101, 175701 (2008); D. Rossini, A. Silva, G. Mussardo, and G. Santoro, ibid. 102, 127204 (2009).

${ }^{7}$ A. Polkovnikov and V. Gritsev, Nat. Phys. 4, 477 (2008).

${ }^{8}$ V. Mukherjee and A. Dutta, J. Stat. Mech.: Theory Exp. (2009), P05005; S. Dutta, S. Lakshmi, and S. K. Pati, Phys. Rev. B 77, 073412 (2008).

${ }^{9}$ Q. Quenching, Annealing and Computation, edited by A. Das, A. K. Chandra, and B. K. Chakrabarti (Springer, New York, 2010), Vol. 802.

${ }^{10}$ W. H. Zurek, Nature (London) 317, 505 (1985); Phys. Rep. 276, 177 (1996); T. W. B. Kibble, J. Phys. A 9, 1387 (1976).

${ }^{11}$ S. Deng et al., EPL 84, 67008 (2008).

${ }^{12}$ S. Miyashita, K. Saito, and H. De Raedt, Phys. Rev. Lett. 80, 1525 (1998).

${ }^{13}$ N. Tsuji, T. Oka, P. Werner, and H. Aoki, arXiv:1008.2594 (unpublished); J. Gong, L. Morales-Molina, and P. Hanggi, Phys. Rev. Lett. 103, 133002 (2009).

${ }^{14}$ M. Lewenstein et al., Adv. Phys. 56, 243 (2007).

${ }^{15}$ B. K. Chakrabarti and M. Acharyya, Rev. Mod. Phys. 71, 847
(1999).

${ }^{16}$ M. Acharyya and B. K. Chakrabarti, Phys. Rev. B 52, 6550 (1995); M. Acharyya, J. K. Bhattacharjee, and B. K. Chakrabarti, Phys. Rev. E 55, 2392 (1997).

${ }^{17}$ T. Tomé and M. J. de Oliveira, Phys. Rev. A 41, 4251 (1990).

${ }^{18}$ A. Kitaev, Ann. Phys. 321, 2 (2006);S. Mandal and N. Surendran, Phys. Rev. B 79, 024426 (2009).

${ }^{19}$ M. Rao, H. R. Krishnamurthy, and R. Pandit, Phys. Rev. B 42, 856 (1990).

${ }^{20}$ S. Ashhab, J. R. Johansson, A. M. Zagoskin, and F. Nori, Phys. Rev. A 75, 063414 (2007).

${ }^{21}$ M. Silverman, Quantum Superposition: Counterintuitive Consequences of Coherence, Entanglement, and Interference (Springer, New York, 2008).

${ }^{22}$ F. Grossmann, T. Dittrich, P. Jung, and P. Hanggi, Phys. Rev. Lett. 67, 516 (1991).

${ }^{23}$ A. Das et al. (unpublished).

${ }^{24}$ V. Mukherjee et al., Phys. Rev. B 77, 214427 (2008).

${ }^{25}$ S. Ghosh et al., Science 296, 2195 (2002).

${ }^{26}$ A. Friedenauer et al., Nat. Phys. 4, 757 (2008).

${ }^{27}$ A. Micheli et al., Nat. Phys. 2, 341 (2006).

${ }^{28}$ L.-M. Duan, E. Demler, and M. D. Lukin, Phys. Rev. Lett. 91, 090402 (2003).

${ }^{29}$ A. Das and B. K. Chakrabarti, Rev. Mod. Phys. 80, 1061 (2008); G. Santoro and E. Tosatti, J. Phys. A 39, R393 (2006); S. Morita and H. Nishimori, J. Math. Phys. 49, 125210 (2008); Quantum Annealing and Related Optimization Methods, edited by A. Das and B. K. Chakrabarti (Springer, Heidelberg, 2005), Vol. 679. 